\title{
Perinatal aspects on the covid-19 pandemic: a practical resource for perinatal-neonatal specialists
}

\author{
Francis Mimouni ${ }^{1,2}$ - Satyan Lakshminrusimha $\mathbb{1}^{3} \cdot$ Stephen A. Pearlman ${ }^{4,5} \cdot$ Tonse Raju $^{6}$ - Patrick G. Gallagher ${ }^{7}$. \\ Joseph Mendlovic ${ }^{8,9}$
}

Received: 23 March 2020 / Revised: 25 March 2020 / Accepted: 25 March 2020 / Published online: 10 April 2020

(c) Springer Nature America, Inc. 2020

\begin{abstract}
Background Little is known about the perinatal aspects of COVID-19.

Objective To summarize available evidence and provide perinatologists/neonatologists with tools for managing their patients. Methods Analysis of available literature on COVID-19 using Medline and Google scholar.

Results From scant data: vertical transmission from maternal infection during the third trimester probably does not occur or likely it occurs very rarely. Consequences of COVID-19 infection among women during early pregnancy remain unknown. We cannot conclude if pregnancy is a risk factor for more severe disease in women with COVID-19. Little is known about disease severity in neonates, and from very few samples, the presence of SARS-CoV-2 has not been documented in human milk. Links to websites of organizations with updated COVID-19 information are provided. Infographics summarize an approach to the pregnant woman or neonate with suspected or confirmed COVID-19.

Conclusions As the pandemic continues, more data will be available that could lead to changes in current knowledge and recommendations.
\end{abstract}

\section{Introduction}

In a near-prophetic study published in 2015, Menachery et al. warned the medical and scientific communities of the

Francis Mimouni

fbmimouni@gmail.com

$\triangle$ Satyan Lakshminrusimha

slakshmi@ucdavis.edu

1 Department of Neonatology, Shaare Zedek Medical Center, Jerusalem, Israel

2 Sackler School of Medicine, Tel Aviv, Israel

3 UC Davis Children's Hospital, Sacramento, CA, USA

4 Christiana Care, Newark, DE, USA

5 Sidney Kimmel Medical College, Thomas Jefferson University, Newark, DE, USA

6 School of Medicine, Uniformed Services University of Health Sciences, Bethesda, MD, USA

7 Yale University School of Medicine, New Haven, CT, USA

8 Shaare Zedek Medical Center, Jerusalem, Israel

9 Recanati Business School, Tel Aviv University, Tel Aviv, Israel continued risk of the reemergence of severe acute respiratory syndrome coronavirus (SARS-CoV) from viruses currently circulating in Chinese bat populations [1]. In the recent past, bat-borne viruses transmitted to humans have led to human to human transmission, with high fatality rates, as happened during the 2002-2003 SARS-CoV-1 [2] and the 2012 Middle East Respiratory Syndrome Coronavirus (MERS-CoV) [3] epidemics.

On November 17, 2019, the first case of infection with a novel coronavirus was identified according to Chinese government sources [4] and the World Health Organization (WHO), which has maintained a day-to-day timeline of the evolution of the current coronavirus pandemic. As predicted by Menachery, through December 2019, sporadic cases in adults from the city of Wuhan, in the Hubei province of China, developed severe respiratory symptoms. Initially, many patients had been exposed at the Hunan wholesale seafood market, but even after closure of this market on January 1, 2020, new cases continued to present at an exponential rate, suggesting human to human transmission $[5,6]$. The outbreak was reported by China to the WHO on December 31 and declared a pandemic by the WHO on March 11, 2020 as many other countries throughout the world reported new cases with high fatality 
rates [5]. These high rates may have been fueled by the rapid propagation of the disease that proved country after country, to rapidly exhaust reserve medical supplies and the ability to provide intensive care to an overwhelming increased numbers of patients [7]. The novel virus was named SARS-CoV-2 and was identified as the agent of a new respiratory syndrome named COVID-19.

Abundant research has been conducted to date in order to better define the epidemiology, clinical characteristics, prevention, and treatment of SARS-CoV-2 infection. Since the first publications on COVID-19 appeared, a Medline search conducted on March 18, using the keyword of COVID-19 retrieved 1087 articles. Yet, very little is known about the perinatal aspects of COVID-19.

The purpose of this Perspective is to summarize the available evidence at this moment, and to provide perinatologists and neonatologists with practical tools for managing their patients, and offering links to reputable medical organizations websites that are frequently updated. We chose to address the following questions:

(1) Is there a vertical transmission of SARS-CoV-2 during pregnancy, and if so, can this lead to abortions, premature labor, or congenital disease such as observed in CMV or Zika disease?

(2) Is pregnancy a risk factor for severe COVID-19?

(3) What are the dangers of SARS-CoV-2 infection in labor?

(4) What are the dangers of SARS-CoV-2 infection in neonates?

(5) Can SARS-CoV-2 be transmitted through human milk?

We will then describe current expert opinions/guidelines of major organizations such as the United States Center for Diseases Control (CDC), the American College of Obstetricians and Gynecologists (ACOG) and the Society for Maternal-Fetal Medicine (SMFM), the United Kingdom National Health Services (NHS), the Royal College of Obstetricians and Gynecologists (RCOG), the Academy of Breastfeeding Medicine (ABM), the National Institutes of Health (NIH), and the American Academy of Pediatrics (AAP).

\section{Is there vertical transmission of SARS-CoV-2 during pregnancy?}

Very few articles have attempted to answer this question. The first was a retrospective review of nine Chinese pregnant women by Chen et al. with laboratory-confirmed COVID-19 pneumonia [8]. All nine patients had a cesarean section in the third trimester, but the indications for this mode of delivery were not clearly stated. Amniotic fluid, cord blood, and neonatal throat swabs all tested negative for the virus.The authors of this small case series suggested that there is currently no evidence for intrauterine infection caused by vertical transmission in women who develop COVID-19 pneumonia in late pregnancy. An additional study published by Zhang in the Chinese language compared 16 pregnant women with COVID-19 to 45 women without COVID-19 [9]. In this study, all infants in both groups were delivered by cesarean section, at a gestational age of $38.7 \pm$ 1.4 weeks in the COVID-19 group and $37.9 \pm 1.6$ weeks in the control group. Only one of the 16 pregnant women with COVID-19 had a severe disease. Ten neonates in the COVID-19 group were tested by PCR and all were found to be negative. Both studies, authored by Chen and Zhang $[8,9]$, came from the same institution and it is not clear whether or not there was an overlap among the patients. An additional Chinese study by Fan et al. [10] described two cases of COVID-19 during the third trimester of pregnancy, which "failed to identify SARS-CoV-2 in all the products of conception and the newborns", and suggested a "low risk of intrauterine infection by vertical transmission of SARSCoV-2". An additional study by Chen et al. [11] reported that three placentas from infected mothers tested negative for the virus. Finally a most recent article by Chen et al. described four infants delivered to mothers with COVID-19 during labor [12]. Infant testing was performed in only three, who all tested negative for the virus. In an update [13], authors of previous series described 33 neonates of whom 3 were reported to have early onset COVID-19 infection. Nasopharyngeal/anal swabs for RT-PCR were not obtained immediately after birth in any of the infants, indicating positivity of the test does not prove vertical transmission. There are only two reports in the nonmedical, general literature that suggested that vertical transmission may be possible. In one report, an infant tested positive but testing took place at $30 \mathrm{~h}$ of age when horizontal transmission cannot be ruled out [14]. In contrast, in the second report, a newborn with a positive test was born to a mother who was also positive for the virus, and the newborn screening took place "within a few minutes" after delivery [15]. This is more suggestive of a vertical transmission. In a recent letter, Dong et al report a newborn with elevated SARS-Cov-2 IgM, IgG and IL-16 levels at 2 hours after birth with negative nasopharyngeal swabs for RT-PCR from $2 \mathrm{~h}$ to 16 $\mathrm{d}$ after birth; amniotic fluid, placenta, or cord blood was not tested [16]. In an accompanying Editorial, Kimberlin and Stagno caution interpretation of these results as confirmation of vertical transmission due to a variety of concerns including technical concerns with IgM assays [17]. Importantly, vertical transmission has not been observed in either SARS-CoV-1 or in MERS-CoV [18]. From these scant data, we cautiously conclude that vertical transmission from 
maternal infection during the third trimester probably does not occur, or likely occurs very rarely. However, the consequences of COVID-19 infection among women during early pregnancy remain unknown. We do not know whether the virus may be vertically transmitted to the fetus causing malformations, fetal growth abnormalities, disruption of maturing fetal organs, or leads to early fetal loss, abortions, or induces premature onset of labor.

\section{Is pregnancy a risk factor for severe SARS- CoV-2 infection?}

Pregnancy is a partially immunocompromised state and any viral or bacterial pneumonia can be life-threatening during pregnancy [19]. However, despite the fact that COVID-19 is known to cause severe life-threatening respiratory complications in adults, especially the immunocompromised, there are no comparative data to determine whether pregnancy is a risk factor for severe SARS-CoV-2 pneumonia. In Chen's study, all nine pregnant women had laboratoryconfirmed SARS-CoV-2. Eight of 9 women had evidence of pneumonia on chest CT [8] and seven presented with fever. Other symptoms included cough among 4 of 9 patients. None of the patients died or developed severe SARS-CoV-2 pneumonia. Based on their small series of nine cases, the authors concluded that the clinical characteristics of COVID-19 pneumonia in pregnant women were similar to those reported for nonpregnant adult patients. In the study by Zhang et al. none of the 16 women with Covid-19 progressed to "critical pneumonia" [9]. Based on these scant data, we cannot conclude if pregnancy is a risk factor for more severe disease in women with COVID-19. It might be important however to determine whether complications of pregnancy such as diabetes, cardiac failure, or hypertension, which have been identified as risk factors for severe COVID-19 infections in nonpregnant adults, also represent additional risk factors for pregnant women [20].

\section{What are the dangers of COVID-19 infections in labor?}

In Chen's study [8], all nine infants born to COVID-19 infected pregnant mothers had normal 1- and 5-min Apgar scores. In Zhang's study [9], the comparison of pregnancy outcomes between 16 women with COVID-19 and 45 women without COVID-19 did not demonstrate any significant differences in fetal distress, meconium-stained amniotic fluid, preterm birth, and neonatal asphyxia. These two small studies, with limited clinical information lack clearly described indications for cesarean deliveries and do not provide an answer to our question.

\section{WHAT are the dangers of SARS-CoV-2 infections in neonates?}

The report by Zhu et al. [21] represents a clinical retrospective analysis of ten neonates born to nine mothers with confirmed COVID-19 pneumonia. There were four full-term infants and six were born prematurely; two were small-for-gestational-age infants and one was large-for-gestational-age (LGA). Six infants developed RDS, two of them were febrile, and two had thrombocytopenia with abnormal liver enzymes. Five infants went home healthy, and when the article was published, four were still hospitalized in stable condition, while one child died. Specimens for SARS-CoV-2 analyses were collected from nine of the ten neonates 1 to 9 days after birth and all were negative. It is unclear if the complications noted were related to prematurity or to SARSCoV-2, but the negative testing would suggest that the virus was unlikely to be the cause. In Dong's report of 2143 children with Covid-19 in China, the illness appeared to be most often less severe than in adults, as only 13 patients were classified as "critical" [22]. In this report, there were no infants classified as neonates, and the youngest ones were classified as less than 1 year old. From this report there is some suggestion that the younger the child, the more severe the disease. In Chen's study [12], four full-term, singleton infants were born to pregnant women who tested positive for COVID-19 in the city of Wuhan where the disease was first identified. None of the infants developed the typical COVID-19 symptoms of fever and cough, however two of them developed rashes, one had facial ulcerations, and one infant had respiratory distress which was supported by noninvasive mechanical ventilation for 3 days. However, none of the three infants out of four infants tested had positive viral results and it is probable that the abovementioned symptoms were unrelated to SARS-CoV-2. In their updated series of 33 infants, Zeng et al [13] describe 3 infants diagnosed with pneumonia with persisting positive viral detection by RT-PCR within a few days of the first PCR; thus these 3 infants probably were truly infected. Whether 2 of 3 term infants diagnosed with pneumonia actually had pneumonia is unclear, as they had no respiratory symptoms and their chest radiographs lacked typical findings of pneumonia. The third infant diagnosed as pneumonia, born prematurely at 31 weeks, suffered from a depressed 5-minute Apgar score, respiratory distress syndrome, and disseminated intravascular coagulation. A blood culture yielded Enterobacter agglomerans, suggesting symptomatology was probably unrelated to SARS-CoV-2. Thus, we cannot define from these limited data the extent of disease severity in neonates. 


\section{Can SARS-CoV-2 be transmitted through human milk?}

In Chen H's study of nine pregnant women with laboratory-confirmed COVID-19 pneumonia, all breast milk samples that were collected and tested from six patients after the first lactation were negative for the virus [6]. This is obviously an insufficient sample size to make a definitive statement that SARS-CoV-2 is or is not excreted in human milk.

\section{Expert opinions/guidelines/useful links}

(1) General prevention measures for preventing the spread of infection:

We recommend using the $\mathrm{CDC}$ site for this purpose. This website is regularly updated, and it is not within the scope of this article to describe these guidelines, which may be found at:

https://www.cdc.gov/coronavirus/2019-ncov/ infection-control/control-recommendations.html.

(2) Guidelines for inpatient obstetric healthcare settings: these guidelines may be found at the website: https://www.cdc.gov/coronavirus/2019-ncov/hcp/inpa tient-obstetric-healthcare-guidance.html.

Briefly, pregnant patients who have confirmed COVID-19 or who are "persons under investigation" (PUIs) are instructed to notify the obstetric unit prior to arrival in order to facilitate appropriate infection control measures. The obstetric unit is to notify infection control personnel in order to obtain appropriate guidance.

Infants born to mothers with confirmed COVID-19 should be considered PUIs and isolated as such.

After birth, in order to reduce the risk of transmission of the virus from the mother to the newborn, facilities should consider temporarily separating mother from her baby in a separate isolation room until the mother's transmission-based precautions are discontinued. Visitors and staff members should use appropriate PPE, including a gown, gloves, facemask, and eye protection. Separation should be discontinued on a case-by-case basis accounting for disease severity, illness signs and symptoms, and results of laboratory testing for the virus.

If rooming-in occurs in accordance with the mother's wishes or is unavoidable due to facility limitations, the newborn should be kept $\geq 6$ feet $(\sim 2 \mathrm{~m})$ away from his or her mother. Should the mother with confirmed COVID-19 or PUI take care of her infant, she should put on a facemask and practice hand hygiene before each feeding or other close contact with her newborn.

(3) Guidelines for breastfeeding.

These guidelines can be found at the website: https://www.cdc.gov/coronavirus/2019-ncov/prepare/ pregnancy-breastfeeding.html.

Briefly: the CDC recommends that during temporary separation, mothers should be encouraged to express their breast milk to establish and maintain milk supply, using a dedicated breast pump, and after adequate hand hygiene. This expressed breast milk is intended may be fed to the newborn by a healthy caregiver. As stated earlier, if a mother and newborn do room-in and the mother wishes to feed at the breast, she should put on a facemask and practice hand hygiene before each feeding.

These CDC recommendations are consistent with the $\mathrm{ABM}$ recommendations to be found in detail at the website:

https://www.bfmed.org/abm-statement-coronavirus.

These are also the basis of the Society for Maternal-Fetal Medicine guidelines to be found at the website:

https://s3.amazonaws.com/cdn.smfm.org/media/ 2267/COVID-19-_updated_3-17-20_PDF.pdf.

ACOG also adopted the CDC guidelines regarding breastfeeding, as can be read in detail at the website:

https://www.acog.org/clinical/clinical-guidance/pra ctice-advisory/articles/2020/03/novel-coronavirus2019.

(4) General advice for pregnant women who may have been exposed to COVID-19 or are experiencing symptoms suggestive of COVID-19. We suggest consulting the UK NHS /RCOGguidelines which recommend that pregnant women concerned about exposure or symptoms indicating possible infection with COVID-19 should not visit their GP practice or attend Emergency Departments in person, but should rather contact their local hotline and tell the operator of possible COVID-19 exposure, and self-isolate until advised otherwise.

More details are provided at the website: https://www.rcog.org.uk/en/guidelines-researchservices/guidelines/coronavirus-pregnancy/covid-19virus-infection-and-pregnancy/.

(5) Advice regarding self-isolation for women with possible or confirmed COVID-19

The above-mentioned NHS guidelines, like others, recommend that pregnant women who have been advised to be self-isolated, should stay indoors and avoid contact with others for 14 days.

(6) The WHO has maintained a day-to-day tally of the global and country-specific information concerning 
Fig. 1 Infographic showing maternal clinical features, possible modes of spread to the neonate and neonatal management of suspected or confirmed COVID-19. There is a wide range of illness among mothers from asymptomatic to pneumonia to severe acute respiratory distress syndrome (ARDS). Term neonates born to COVID-19 mothers are usually asymptomatic. PPE personal protective equipment, CPAP continuous positive airway pressure, HFNC high flow nasal cannula, PAPR powered air purifying respirator.
SUSPECTED OR CONFIRMED COVID-19 DURING PREGNANCY: MATERNAL CLINICAL FEATURES, DELIVERY ROOM PRECAUTIONS AND NEONATAL MANAGEMENT

Specially prepared delivery room or operating room preferably with negative pressure Obstetric and neonatal providers with full PPE

Droplet and contact precautions: Gown, glove, mask and eye shield

Neonatal resuscitation as per current guidelines

Airborne precautions - especially with suction, intubation, CPAP and other aerosol generating procedures

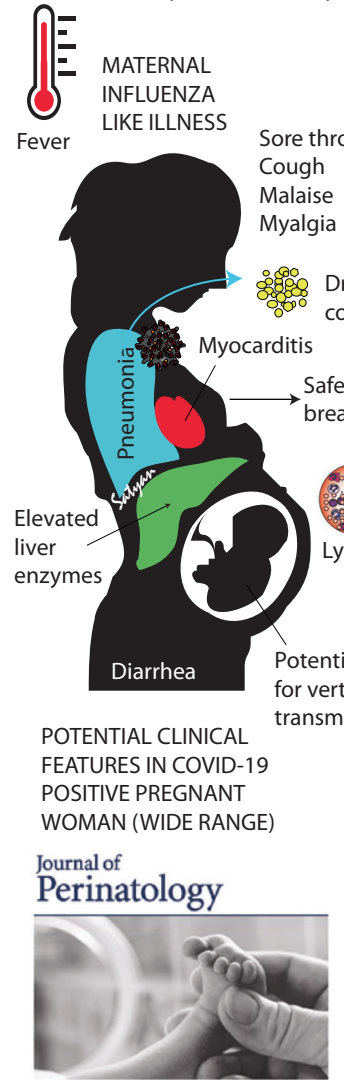

DELIVERY MODE:
Dictated by usual obstetric practice

MODES OF TRANSMISSION

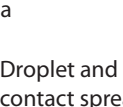
tact spread

$\int$ Extu Aerosol generating procedures: Suction Intubation HFNC $\longrightarrow$ N-CPAP $\quad$ P $\quad \begin{aligned} & \text { precautions - PPE } \\ & \text { including eye shield }\end{aligned}$ and $\mathrm{N} 95$ mask or PAPR, gown and gloves for delivery room personnel

NEONATAL MANAGEMENT

Consider temporarily separating the infant from the mother;

Healthy caregiver may feed expressed breast milk;

If mother and infant are not separated, breastfeeding while wearing a mask and after hand hygiene may be considered;

NEONATES - MOSTLY ASYMPTOMATIC

\section{(non-specific symptoms} such as temperature instability, apnea, respiratory for RT-PCR at $24 \mathrm{~h}$ and repeat distress, Gl symptoms, hypotension are possible) $\mathrm{X} 1$ at $48 \mathrm{~h}$;

Isolation pending test results;

NEWBORN DISCHARGE - as per clinical status; transmission precautions at home based on infant's test results statistics and public health measures undertaken at individual country levels.

https://www.who.int/emergencies/diseases/novelcoronavirus-2019.

(7) The NIH has provided a quick access to the most recent research on COVID-19 at the following address:

https://www.nih.gov/health-information/coronavirus.

This address allows registering to a mailing list of participants that will receive automatic updates from the NIH on Coronavirus.

(8) The AAP has built an educational website on COVID19, that may be reached at the following address: https:// services.aap.org/en/pages/2019-novel-coronaviruscovid-19-infections/. Within this website, one can read a document of "Initial Guidance" on the management of infants born to mothers with COVID-19, published on April 2, 2020, that may be downloaded from the following address: https://download.aap.org/AAP/PDF/ COVID\%2019\%20Initial\%Newborn\%20Guidance.pdf.
This document is particularly useful as it describes the personal protective equipment to be used and isolation precautions to be taken, both for customary contact and for high risk situations such as when patients require bag and mask ventilation, intubation, tracheal suctioning, nasal cannula oxygen at high flow, continuous positive airway pressure and/or positive pressure ventilation of any type, given the potential for these supports to generate aerosols. It also provides preliminary guidelines for delivery room management of neonates, instructions for newborn admission after maternal separation, and guidance for alternative well newborn care. The AAP recommends at this point in time that molecular assay testing should be done at approximately 24 hours of age in all infants born to mothers with COVID-19, and that repeat testing should be done at approximately 48 hours of age. The AAP document also addresses the issue of maternal visitation for infants requiring ongoing hospital care, and spells the criteria that need to be met for visitation to happen. 


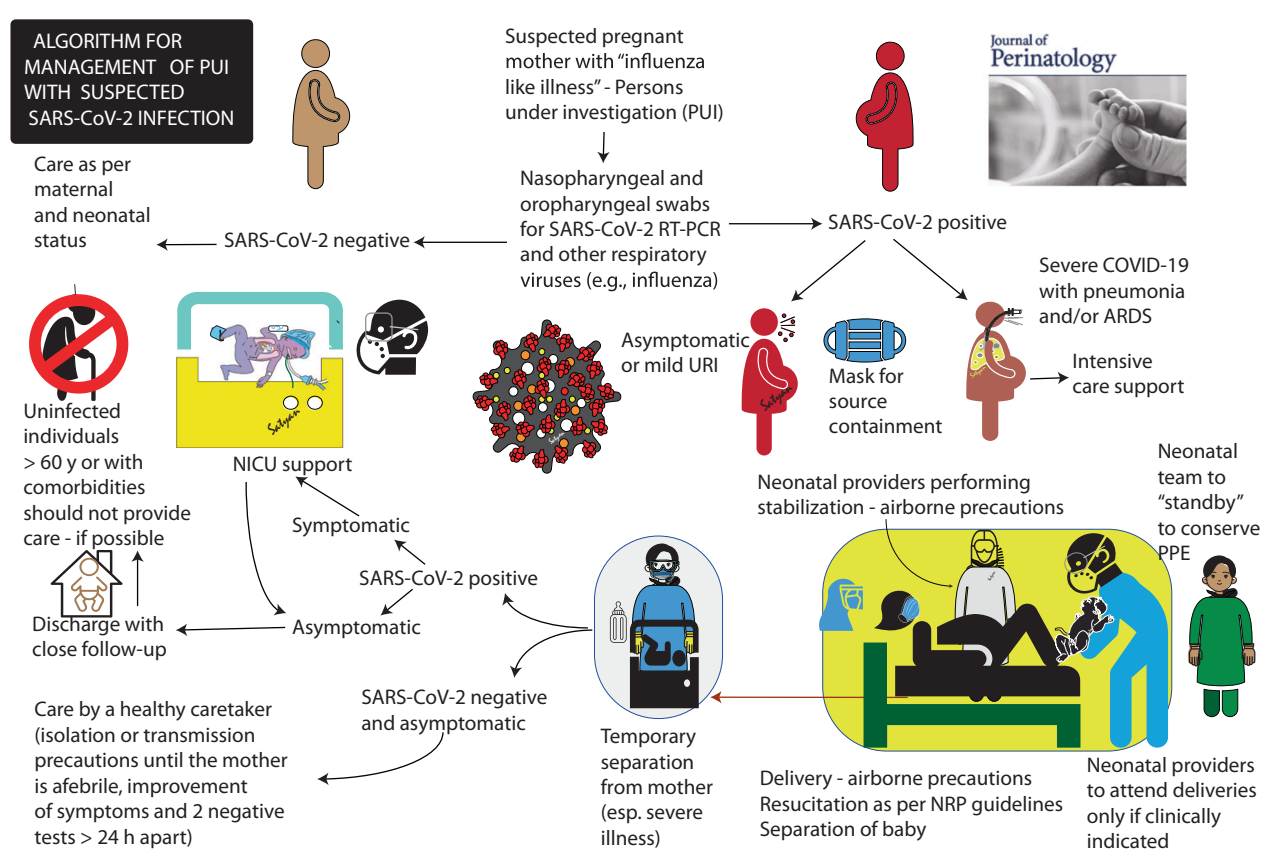

Fig. 2 Current recommended management of suspected pregnant woman with influenza-like illness with suspected novel 2019 coronavirus infection (COVID-19). If maternal tests are positive, mothers should wear a mask for source containment. Minimum number of providers should be in the delivery/operating room to reduce exposure risk. Additional providers can be on standby outside the room to reduce personal protective equipment (PPE) burnout. Neonatal providers performing infant stabilization should attend delivery with PPE to provide airborne precautions and infant is immediately isolated from the mother after delivery and cared in a

\section{Conclusion}

We strongly advise the readers that this Perspective be considered preliminary. As the pandemic continues, there will be more data available that could lead to significant changes in the current recommendations. The abovementioned websites have been built by reputable organizations and are updated frequently. They should establish the best perinatal and neonatal practices. In the meanwhile, we are providing an illustration (Fig. 1) that summarizes many of the concepts described in this text. Figure 2 outlines a suggested algorithm for the perinatal and neonatal management of a PUI (person under investigation) with suspected COVID-19 infection.

Author contributions FM and JM wrote the paper, SP and SL edited paper, SL prepared the artwork, and TR and PG contributed to the concept, scientific content, and editing of the paper.

\section{Compliance with ethical standards}

Conflict of interest The authors declare that they have no conflict of interest. separate room pending testing. Recommended testing is nasopharyngeal and throat swabs at $24 \mathrm{~h}$ after birth and repeated $48 \mathrm{~h}$ after birth. If these tests are negative and baby is asymptomatic, he/she can be discharged to be cared for by a noninfected, asymptomatic caretaker pending negative repeat tests and resolution of symptoms in the mother. If infant is positive but asymptomatic, baby can be discharged but may need to be quarantined at home. If possible, uninfected individuals $>60$ years of age or with comorbid conditions should not care for these infants. If symptomatic, baby needs NICU care. These guidelines are subject to change with emerging evidence.

Publisher's note Springer Nature remains neutral with regard to jurisdictional claims in published maps and institutional affiliations.

\section{References}

1. Menachery VD, Yount BL Jr, Debbink K, Agnihothram S, Gralinski LE, Plante JA, et al. A SARS-like cluster of circulating bat coronaviruses shows potential for human emergence. Nat Med. 2015;21:1508-13.

2. Chan-Yeung M, Xu RH. SARS: epidemiology. Respirology. 2003;8:S9-14.

3. Middle East Respiratory Syndrome Coronavirus. 2020. https://www.who.int/emergencies/mers-cov/en/. Accessed 16 Feb 2020.

4. "The first COVID-19 case originated on November 17, according to Chinese officials searching for 'Patient Zero'“. 2020. www. msn.com.

5. Singhal T. A review of coronavirus disease-2019 (COVID-19). Indian J Pediatr. https://doi.org/10.1007/s12098-020-03263-6. 2020. In press.

6. Huang C, Wang Y, Li X, Ren L, Zhao J, Hu Y, et al. Clinical features of patients infected with 2019 novel coronavirus in Wuhan, China. Lancet. 2020;395:497-506.

7. Wang X, Zhang X, He J. Challenges to the system of reserve medical supplies for public health emergencies: reflections on the 
outbreak of the severe acute respiratory syndrome coronavirus 2 (SARS-CoV-2) epidemic in China. Biosci Trends. 2020;14:3-8.

8. Chen H, Guo J, Wang C, Luo F, Yu X, Zhang W, et al. Clinical characteristics and intrauterine vertical transmission potential of COVID-19 infection in nine pregnant women: a retrospective review of medical records. Lancet. 2020;395:809-15.

9. Zhang L, Jiang Y, Wei M, Cheng BH, Zhou XC, Li J, et al. Analysis of the pregnancy outcomes in pregnant women with COVID-19 in Hubei Province. Zhonghua Fu Chan Ke Za Zhi. 2020;55:E009.

10. Fan C, Lei D, Fang C, Li C, Wang M, Liu Y, et al. Perinatal transmission of COVID-19 associated SARS-CoV-2: Should we worry? Clin Infect Dis. 2020. In press. https://doi.org/10.1093/cid/ciaa226.

11. Chen S, Huang B, Luo DJ, et al. Pregnant women with new coronavirus infection: a clinical characteristics and placental pathological analysis of three cases. Zhonghua Bing Li Xue Za Zhi. 2020;49:E005.

12. Chen y, Peng H, Wang L, Zhao Y, Zeng L, Gao H, et al. Infants born to mothers with a new coronavirus (COVID-19). Front Pediatr. 2020. In press. https://doi.org/10.3389/fped.2020.00104.

13. Zeng L, Xia S, Yuan W, Yan K, Xiao F, Shao J, et al. Neonatal early-onset infection with SARS-CoV-2 in 33 neonates born to mothers with COVID-19 in Wuhan, China. JAMA Pediatrics. https://doi.org/10.1001/jamapediatrics.2020.0878. 2020. In press.

14. D'Amore R. Can coronavirus pass from mother to baby? Maybe, but experts need more research. Global News. 2020. https://globa lnews.ca/news/6515302/coronavirus-mother-babytransmission/. Accessed 10 Feb 2020.
15. Murphy S. Newborn baby tests positive for coronavirus in London. The Guardian. 2020: https://www.theguardian.com/world/ 2020/mar/14/newborn-baby-tests-positive-for-coronavirus-inlondon.

16. Dong L, Tian J, He S, Zhu C, Wang J, Liu C et al. Possible vertical transmission of SARS-COVID-2 from an infected mother to her newborn. JAMA. https://doi.org/10.1001/jama.2020.46211. 2020. In press.

17. Kimberlin DW, Stagno S. Can SARS-CoV-2 infection be acquired in utero?: More definitive vevidence is needed. JAMA. https://doi.org/10.1001/jama.2020.4868. 2020. In press.

18. Rasmussen S, Smulian J, Lednicky J, et al. Coronavirus disease 2019 (COVID-19) and pregnancy: what obstetricians need to know. Am J Obstet Gynecol. 2020. In press. https://doi.org/10. 1016/j.ajog.2020.02.017.

19. Mathad JS, Gupta A. Pulmonary infections in pregnancy. Semin Respir Crit Care Med. 2017;38:174-84.

20. Li B, Yang J, Zhao F, Zhi L, Wang X, Liu L, et al. Prevalence and impact of cardiovascular metabolic diseases on COVID-19 in China. Clin Res Cardiol. 2020. In press. https://doi.org/10.1007/ s00392-020-01626-9.

21. Zhu H, Wang L, Fang C, Peng S, Zhang L, Chang G, et al. Clinical analysis of 10 neonates born to mothers with 2019-nCoV pneumonia. Transl Pediatr. 2020;9:51-60.

22. Dong Y, Mo X, Hu Y, Qi X, Jiang F, Jiang Z, et al. Epidemiological characteristics of 2143 pediatric patients with 2019 coronavirus disease in China. Pediatrics. 2020. In press. https://doi. org/10.1542/peds.2020-0702. 